Revista Venezolana de Gerencia (RVG)

Año 16. N 56, 2011, 608 - 621

Universidad del Zulia (LUZ) • ISSN 1315-9984

\title{
Valor razonable como método de medición de la información financiera
}

\author{
Gómez, Oda* \\ De La Hoz, Betty ** \\ López, Margo***
}

\section{Resumen}

Partiendo de la premisa que la información financiera presentada a su valor razonable refleja la situación real de la empresa, se realizó esta investigación exploratoria, de tipo documental, ubicada en el área de la contabilidad financiera, cuyo objetivo fue sistematizar las bases teóricas que respaldan la fiabilidad del valor razonable contempladas en las normas de contabilidad emitidas por el Financial Accounting Standards Board, International Accounting Standards Board, y las normas de contabilidad aplicadas en Venezuela. De dicha revisión se derivo que su aplicación, a diferencia de otros métodos, facilita la comparabilidad de la información financiera en cualquier contexto económi$\mathrm{co}$, sea nacional o internacional, eliminando las distorsiones potenciales que limitan la toma de decisiones. Se concluye que el uso del valor razonable produjo un cambio de paradigma en la contabilidad financiera dando prioridad a la esencia financiera sobre la forma legal; que la gerencia de las organizaciones como responsables de la cuantificación y revelación de la información financiera a valor razonable, requiere entender el concepto de valor razonable y todas las disposiciones establecidas en la norma internacional de información financiera número trece- Medición del Valor Razonable, con el propósito de garantizar la fiabilidad de la información financiera.

Palabras clave: Valor razonable, normas internacionales de contabilidad, normas internacionales de información financiera.

Recibido: 03-11-10 Aceptado: 11-08-11

* Licenciada en Contaduría Pública. Magister en Gerencia de Empresas. Mención: Finanzas. Profesora titular de la Escuela de Administración y Contaduría Pública y del Post-Grado en Gerencia de Empresas de la Facultad de Ciencias Económicas y Sociales de la Universidad del Zulia. (LUZ).e-amil: odagomez@cantv.net

** Licenciada en Contaduría Pública. Magíster Scientiarum en Gerencia de Empresas. Mención: Gerencia Financiera. Docente de la Universidad del Zulia (LUZ) y de la Universidad Rafael Urdaneta (URU), Investigadora adscrita al Instituto de Investigaciones y al Departamento de Contabilidad de la Facultad de Ciencias Económicas y Sociales de la Universidad del Zulia. e-mail: betty.hoz@hotmail.com

*** Licenciada en Administración de Empresas. Magister Scientiarum en Gerencia de Empresas. Escuela de Negocios. Docente de la Universidad Rafael Urdaneta y Universidad del Zulia. Maracaibo. Estado Zulia. Venezuela. e-mail: margolopez@hotmail.com 


\title{
Reasonable Value as a Method for Measuring Financial Information
}

\begin{abstract}
Starting from the premise that financial information presented at its reasonable value reflects the real situation of an enterprise, exploratory research of a documentary nature was carried out in the financial accounting area whose objective was to systematize the theoretical bases that support the reliability of reasonable value contemplated in the accounting standards issued by the Financial Accounting Standards Board, International Accounting Standards Board and the accounting standards applied in Venezuela. From this review of the literature, it was seen that its application, as opposed to other methods, facilitates the comparability of financial information in any economic context, whether national or international, eliminating the potential distortions that limit decision making. Conclusions are that the use of reasonable value produces a paradigm shift in financial accounting, giving priority to the financial essence over and above the legal form; that organizational management, which is responsible for quantifying and revealing the financial information at its reasonable value, needs to understand the concept of reasonable value and all the dispositions established in the international standard for financial information, number thirteen - Measurement of Reasonable Value - in order to guarantee the reliability of the financial information.
\end{abstract}

key words. Reasonable value, international accounting standards, international financial information standards.

\section{Introducción}

La contabilidad como disciplina científica nació como una necesidad social de medición de la realidad económica, al cuantificar las transacciones que realizan las organizaciones; siendo el proceso medición una de las etapas fundamentales en la elaboración de la información financiera de las empresas. Durante mucho tiempo se utilizó como método de medición el valor histórico; sin embargo, la necesidad de información financiera sobre el valor real del negocio, ha originado la incorporación de otros métodos, como son: costo corriente, valor realizable, valor presente, valor razonable, entre otros; para medir los elementos (activo, pasivo, patrimonio neto, ingresos y egresos) de los estados financieros.
Una de las críticas a la información financiera es que no refleja la situación financiera real de la organización, originando a través del tiempo la incorporación de nuevos métodos de medición, siendo el más reciente el valor razonable que se aplica a algunas partidas de los elementos de los estados financiero, dando respuesta a la necesidad de los usuarios de recibir información real y oportuna sobre las actividades económicas y sociales realizadas por la organización.

La mayoría de los modelos contables aplicados en los distintos países por varios siglos fue el método de medición a costo histórico, en algunos casos ajustados por efectos de la inflación, para reflejar la situación económica del país. Con las normas Stament of Financial Accounting Standards (SFAS), las normas internacionales de contabilidad (NIC) y las 
Valor Razonable como método de medición de la Información Financiera Gómez, Oda; De La Hoz, Betty y López, Margo

Normas Internacionales de Información Financiera (NIIF ${ }^{1}$ ), se ha incrementado la aplicación del método de medición a valor razonable.

Recientemente con la crisis financiera del año 2008, producto de las hipotecas subprime que se originó en los Estados Unidos afectando el sistema financiero internacional, se puso en debate la fiabilidad del valor razonable y su influencia en la evolución de los mercados financieros.

Por esta razon se considero pertinente explorar las bases teóricas que respaldan la fiabilidad del valor razonable establecidas en la normativa contable. Esta investigación corresponde al área del conocimiento de la contabilidad financiera, siendo esta la disciplina que se encarga de estudiar el proceso de medir las actividades económicas - sociales de una organización, que se resumen y presentan a los usuarios internos y externos en forma de estados financieros, para tomar decisiones económicas.

\section{Antecedentes del valor razonable}

Desde el siglo XV la contabilidad se ha venido apoyando, para fines de uniformidad, en principios, convenciones, postulados y normas. Hasta los años setenta el criterio básico de valoración, era el costo histórico porque además de conservador y objetivo, los conceptos de valores corrientes y de volatilidad, eran más académicos que reales.

Es importante aclarar que esta investigación está orientada al valor razonable establecido en las normas de contabilidad del denominado "Fair Value" o Valor Razonable, emitidas por la Finalcial Accounting Standards Boards (FASB) y el Internacional Acco Unting Standards Boards (IASB).

Según Fortis y García (2006), durante los años 80 , los instrumentos financieros derivados experimentaron un importante desarrollo al ser utilizados como cobertura de los riesgos de tipo de interés y tipo de cambio. Esto más la importancia cada vez mayor de los mercados de capitales como fuentes de inversión y financiamiento, exigen que la información financiera sea representación fiel del valor de la organización.

El país que primero se enfocó sobre el valor razonable fue los Estados Unidos de América con la aparición en 1992, de las normas Statement of Financial Accounting Standards (SFAS 107), donde la FASB, exigía publicar el valor razonable de todos los instrumentos financieros. Además el SFAS 115 indicaba que el valor razonable de algunos valores negociables se incluyera en el balance general y en las cuentas de resultados. $A$ pesar de que dichas normas fueron criticadas en el año 1996, en el Reino Unido el regulador contable (Accounting Standards Board) publicó un documento en el

1 Las Normas Internacionales de Contabilidad se emitieron inicialmente con las siglas en español NIC, en el año 2001 el organismo emisor fue reestructurado, y partir de ese año decidió emitir las Normas Internacionales de Información Financiera (NIIF), adoptando las NIC. 
que indicaba que el modelo mixto no era satisfactorio, recomendando la valoración de todos los instrumentos financieros a valor razonable.

Por otro lado, Fortis y García (2006) indican que el modelo "full fair value" se apoya también en las críticas que pueden hacerse al modelo mixto de valoración; modelo donde algunos instrumentos se registran a su costo histórico y otros se registran a valor razonable. En el modelo mixto, los criterios para valorar un instrumento a su costo o valor de mercado no dependen de las características del instrumento sino que se apoyan principalmente en la intención por parte de la entidad de mantenerlo a largo plazo o utilizarlo para la negociación.

En el año 1999, el Comité de Normas Internacionales de Contabilidad (IASC), más tarde sustituido por el Consejo de Normas Internacionales de Contabilidad (IASB), emitió una norma de contabilidad identificada como NIC 39 Instrumentos financieros: reconocimiento y medición, la cual exigía el uso del valor razonable con fines de negociación. Posteriormente en agosto del 2001, el IASB anunció un proyecto de modificación de esta norma, publicándose en diciembre del 2003.

Inicialmente las normas internacionales de contabilidad que se emitieron requerían del costo histórico y producto de la dinámica en la actividad económicasocial donde se desarrollan las organizaciones se fueron incorporando de manera creciente las mediciones y revelaciones a

Siglas en ingles de Proyecto de Norma valor razonable. Es relevante mencionar que el valor razonable no elimina el método de medición a costo histórico, pero si le da un contexto diferente, porque se requiere de revisiones anuales.

García y Zorio (2002), afirman que el valor razonable, viene a formar parte integral de varias normas internacionales tales como la NIC 16, que introduce el valor razonable para las permutas de activos no similares; la NIC 17 sobre arrendamientos explica que tales gastos deben reconocerse como activos y pasivos al menor de los valores razonables; la NIC 18 prescribe que los ingresos deben medirse al valor razonable; la NIC 19 exige que la medición en los beneficios para los empleados sea la diferencia entre el valor presente y el valor razonable; la NIC 20 expresa la medición a valor razonable de las subvenciones gubernamentales; la NIC 39, aborda lo referente al valor razonable en los instrumentos financieros; la NIC 40 supone alternativas opcionales para el valor inmobiliario y la NIC 41 de irrestricta exige la valoración de los activos biológicos a valores razonables.

En septiembre de 2006 el Financial Accounting Standards Board emitió SFAS 157 Fair Value Measurements, donde se define el valor razonable y amplia las revelaciones acerca de las mediciones a valor razonable, y en mayo de 2009 el IASB emitió el borrador para discusión pública (ED) ${ }^{2}$ ED/2009/5 Fair Value Measurement (medición hecha a valor razonable), cuyos conceptos se pre- 
Valor Razonable como método de medición de la Información Financiera Gómez, Oda; De La Hoz, Betty y López, Margo

sentan en la sección del concepto de valor razonable.

Finalmente en mayo de 2011 se materializa el proyecto de valor razonable, cuando el IASB emite la NIIF $13^{3}$ Medición del valor razonable, completando uno de los proyectos del proceso de convergencia entre el IASB y FASB, lo cual constituye un elemento importante de respuesta de dichos organismos a la crisis financiera mundial. Dicha norma es aplicable para los períodos de presentación de la información financiera que comiencen a partir del 1 de enero de 2013, se permite la aplicación anticipada.

El objetivo de la NIIF 13 es aumentar la coherencia y la comparabilidad en las mediciones de valor razonable y revelaciones relacionadas a través de la misma jerarquía establecida en el proyecto, siendo más explícita en las orientaciones dadas para determinar el valor razonable. Esta norma no amplía el uso del valor razonable, pero proporciona orientación sobre cómo debe aplicarse en caso de que su uso sea requerido o permitido por otras normas dentro de las NIIF.

Esta nueva norma requiere de estudio y asimilación por parte de la gerencia de las organizaciones, como responsable de los cálculos y revelaciones del valor razonable presentados en la información financiera; quién debe garantizar el cumplimiento de la fiabilidad de la información financiera.

También será un campo de estudio para los investigadores, con el propósito de verificar si la misma da orientaciones suficientes a los preparadores de la información financiera y los usuarios para comprender mejor las mediciones del valor razonable.

\section{Valor razonable: una aproximación conceptual}

De acuerdo Mantilla (2009), el concepto de valor razonable tiene más de cincuenta años de aplicación en los negocios, principalmente los que se realizan en o alrededor de los mercados de capitales, generalmente se asocia con los precios de mercado y también se le conoce como el valor justo y transparente. Este método de medición nace como una necesidad a los cuestionamientos que se le hacen a la contabilidad medida a costo histórico, por las discrepancias entre el valor de mercado y el valor en libros.

Según Ryan, Scapens y Theobald (2004), dos corrientes doctrinales se han impuesto en los últimos treinta años, la doctrina del Financial Accounting Standards Board (FASB) de los Estados Unidos, de enorme influencia en los profesionales de las compañías de auditoría en todo el mundo; y las normas del International Accounting Standards Board (IASB), organismo emisor de las NICNIIF con vocación universal. Las normas de contabilidad de la FASB están basadas en reglas y las del IASB en principios.

En septiembre de 2006, la FASB, emitió el FASB Statement No. 157, Fair Value Measurements (mediciones hechas a valor razonable) (SFAS 157), don- 
de se establece el marco de referencia para medir el valor razonable y define el valor razonable como: "el precio que se recibiría al vender un activo o que se pagaría al transferir un pasivo en una transacción regular entre los participantes del mercado en la fecha de medición". Lo más resaltante de esta definición es que pone énfasis en que el objetivo de la medición del valor razonable es un precio de salida desde la perspectiva de un participante del mercado que controla el activo o adeuda el pasivo; además aclara que el precio se fija bajo la óptica hipotética en la fecha de medición.

EI SFAS 157, establece una jerarquía de tres niveles, con base en el tipo de datos de entrada utilizadas en las técnicas de medición:

- Los datos de entrada del nivel 1, son los precios cotizados en mercados activos para partidas idénticas el activo o el pasivo que se mide, ejemplo las acciones que se cotizan en la bolsa de valores.

- Los datos de entrada del nivel 2, son otros valores de entrada observables, ejemplo un swap de tasa de interés.

- Los datos de entrada del nivel 3, son valores de entrada no observables pero que deben desarrollarse para reflejar los supuestos que los participantes del mercado utilizarían cuando determinen un precio adecuado para el activo o el pasivo, ejemplo un instrumento de capital o deuda de una entidad privada.

Posteriormente en febrero 2007, emitió el FASB Statement No. 159, The Fair Value Option for Financial Assets and Financial Liabilities (la opción del valor ra- zonable para activos financieros y pasivos financieros), esta norma incluye una enmienda del FASB Statement No. 115.

Deloitte (2008) menciona que esta norma tiene como propósito incrementar la consistencia, la comparabilidad y la transparencia de las mediciones hechas a valor razonable utilizadas en la información financiera, ya que establece: una sola definición con carácter de autoridad, para el valor razonable (precio de salida), una estructura para la medición de valor razonable y amplia los requerimientos de revelación del valor razonable.

De acuerdo a un estudio realizado por Deloitte (2008) a un grupo de 50 entidades registradas en la Securities and Exchge Commision (SEC) del sector bancario, seguros, inversión y energía, se observó que la mayoría de las compañías no reportaron ajustes de transición importantes para las ganancias retenidas ya sea por el SFAS 157 o el SFAS 159, y la composición de las mediciones hechas a valor razonable, dentro de la jerarquía del valor razonable, varió significativamente por industria. Los resultados de esta investigación reflejan que los niveles de jerarquía establecidos en esta norma eran necesarios para poder mostrar el valor razonable de acuerdo las circunstancias del mercado.

Con la aplicación del SFAS 157 se requiere que las organizaciones midan el valor razonable usando los supuestos que se espera sean utilizados por otros participantes en el mercado, incluyendo los supuestos de riesgo y la maximización del los insumos observables

De acuerdo al IASB (2009), el concepto de valor razonable que aparece en 
Valor Razonable como método de medición de la Información Financiera Gómez, Oda; De La Hoz, Betty y López, Margo

las NIC-NIIF "es el importe por el cual puede ser intercambiado un activo, o cancelado un pasivo, entre un comprador y un vendedor interesado y debidamente informados, que realizan una transacción libre". Como se puede observar no hay discrepancias relevantes entre este concepto y el emitido por la FASB.

Respecto a la confiabilidad o fiabilidad del valor razonable, Fortis y García (2006), indican que la información contable es fiable cuando muestra fielmente la realidad económica de la transacción, independientemente de su forma legal, además de ser prudente, completa y libre de distorsiones.

Producto de la crisis financiera del año 2008, por los problemas derivados de la crisis crediticia en los Estados Unidos, el Congreso de ese país de acuerdo a lo dispuesto por la Emergency Economic Stabilization y algunos representantes de las instituciones financieras recomendaron a los organismos internacionales de emisores de normas contables y reguladores, la suspensión o eliminación del valor razonable.

Ante la situación planteada, la SEC (2008) entregó el reporte requerido por el Congreso de los EE.UU. que aborda los siguientes asuntos críticos: los efectos de tales normas contables en el balance de situación; los impactos de la contabilización en la quiebra de bancos en el año 2008; el impacto de dichas normas en la calidad de la información financiera disponible para los inversores; el proceso seguido por el Financial Accounting Standards Board al desarrollar normas contables; la oportunidad y la flexibilidad de tales normas; y las normas contables alter- nativas a las previstas en el Statement 157 (Fair Value Measurements).

Los resultados de este informe indican que los inversores consideran que la contabilización a valor razonable mejora la transparencia de la información financiera, facilitando información confiable para la toma de decisiones, también menciona que este método de medición no es la causa fundamental en la quiebra de los bancos acaecida en el año 2008; indicando que las quiebras bancarias en EE.UU. parecía ser el resultado de unas crecientes pérdidas probables de crédito, preocupaciones sobre la calidad de los activos y en algunos casos, excesiva confianza de los prestamistas e inversores. Dicho informe no recomienda suspender las actuales normas contables sobre el valor razonable, formula recomendaciones para mejorar su aplicación, incluyendo:

- El desarrollo de guías adicionales y otras herramientas para determinar el valor razonable cuando la información relevante del mercado no sea disponible en mercados ilíquidos o inactivos.

- El fortalecimiento de las actuales normas de revelación y presentación relacionadas con el valor razonable en los estados financieros.

- Los esfuerzos formativos, incluyendo los dirigidos a fortalecer la necesidad de criterio para los gestores al determinar las estimaciones de valor razonable.

- El análisis por el FASB del impacto de la liquidez en la valoración del valor razonable, incluyendo si se garantiza la aplicación adicional y/o las guías de revelación. 
- La valoración por el FASB de si la incorporación del riesgo de crédito en la valoración de los pasivos aporta información útil a los inversores.

- Aconseja que la FASB valore de nuevo los modelos actuales de deterioro, incluyendo la posibilidad de reducir el número de modelos bajo las US GAAP $^{4}$

En mayo de 2009, el IASB emitió el borrador para discusión pública $\left(E D^{5}\right)$ ED/2009/5 Fair Value Measurement (medición hecha a valor razonable), con el objeto de: establecer una guía para la determinación del valor razonable, reduciendo la complejidad y mejorando la consistencia en la aplicación de dicho concepto; clarificar la definición del valor razonable y redactar las guías para su aplicación en forma clara; dando una definición simple y unificada sobre el valor razonable que será ampliada con una guía especial para su aplicación en los mercados ilíquidos, está acorde con la solicitud del G- $20^{6}$, de alinear la medición del valor razonable según las NIC-NIIF y las US GAAP.

El proyecto define el valor razonable como "el precio que sería recibido por vender un activo o pagado para transferir un pasivo en una transacción ordenada, no forzada entre los participantes en el mercado a la fecha de medición". Esto aplicaría a todas las mediciones o revelaciones hechas contenidas en las NIC-NIIF, siendo necesario que la entidad determine lo siguiente: el activo o pasivo que este sujeto a la medición; la premisa de evaluación para el activo que sea apropiada para la medición; el mercado más ventajoso para el activo y pasivo; la(s) técni$\mathrm{ca}(\mathrm{s})$ de valuación que sea(n) apropiada(s) para la medición, teniendo en consideración la disponibilidades de datos a partir de los cuales desarrollar los insumos que representen los supuestos que los participantes en el mercado usarían al fijar el precio del activo o pasivo, así como el nivel de jerarquía.

Las limitaciones de la aplicación del valor razonable se presentan cuando no existe un mercado activo ${ }^{7}$ para ciertos activos y pasivos; ya que los mismos forman parte de la estructura fija de la empresa, permanecen en el patrimonio hasta su amortización o vencimiento, siendo difícil determinar su valor.

El proyecto establece que las técnicas de valuación deben ser: aplicadas de manera consistente, maximizar el uso del los insumos observables que sean rele-

$4 \quad$ Normas de contabilidad de los EE.UU.

$5 \quad$ Siglas en inglés del borrador para discusión pública

6 Son 19 países más 1 representante por la Unión Europea, a saber Alemania, Arabia Saudí, Argentina, Australia, Brasil, Canadá, China, Estados Unidos, Francia, India, Indonesia, Italia, Japón, México, República de Corea, Reino Unido, Rusia, Sudáfrica, Turquía y la Unión Europea. ( 8 países más industrializados o sea el G8, más 11 países emergentes, más 1 representante por la Unión Europea).

7 a) Los bienes o servicios intercambiados en el mercado son homogéneos; b)Pueden encontrarse en todo momento compradores o vendedores para un determinado bien o servicio; c)Los precios son conocidos y fácilmente accesibles para el publico 
Valor Razonable como método de medición de la Información Financiera Gómez, Oda; De La Hoz, Betty y López, Margo

vantes y debe ser evaluada periódicamente, bajo los enfoques de mercado, ingresos y costos que se describen a continuación.

El enfoque de mercado utiliza precios y otra información relevante generada por las transacciones de mercado que involucran activos y pasivos idénticos o comparables. Este enfoque parte del supuesto que el valor razonable puede estimarse, por medio del análisis de precios negociados, los cuales se observan en cualquier mercado de activos y pasivos comparables.

El enfoque de ingresos es una técnica de valuación utilizada para convertir importes futuros a un valor presente único. La medición se basa en el valor indicado por las expectativas actuales del mercado sobre dichos importes futuros.

El enfoque de costos que refleja la cantidad que actualmente se requeriría para reemplazar la capacidad de servicio del activo y se basa en el principio de sustitución. Este enfoque se sustenta en la noción de que un participante de mercado no pagaría por un activo más del monto necesario para reemplazar el activo.

Finalmente, este anteproyecto se materializó en mayo de 2011, cuando IASB emite la NIIF-13 Medición del Valor Razonable, donde se ofrece una fuente de orientación única que explica cómo medir el valor razonable, y está en línea con la aplicación establecida por la FASB, de una jerarquía única de tres niveles para todas las mediciones del valor razonable.

La generalización del concepto de valor razonable ha supuesto un cambio con respecto a los principios clásicos del sistema contable basados en la fiabilidad, lo cual ha supuesto algunas ventajas, tales como el acercamiento del resultado contable al económico, lo que se considera una consecuencia positiva para un funcionamiento eficiente del mercado y para el uso de la información contable en la valoración de empresas.

Según Trombetta (2009) el valor razonable estará en la agenda de los investigadores por muchos años, ya que existe la idea que la aplicación del valor razonable generara beneficio en la presentación de la información financiera; sin embargo, en el mundo académico hay dudas respecto a sus beneficios y no se puede afirmar que con la aplicación del valor razonable la información financiera es fiable.

Con la aplicación del método de medición del valor razonable, se origina un cambio claro de la disciplina contable, de un principio legalista de prudencia se pasa a un principio de esencialidad, también conocido como principio de realidad económica o sustancia sobre forma.

\section{Valor razonable en las normas de contabilidad aplicadas en Venezuela}

Según la Declaración de Principios de Contabilidad $\mathrm{N}^{\circ} 0$ (DPC-0) emitida por la Federación de Colegio de Contadores Públicos de Venezuela (1997), en Venezuela desde el año 1997 por efecto supletorio de las normas nacionales se deben estar aplicando más del $50 \%$ de las NICNIIF emitidas por IASB. Adicionalmente según Marco de Adopción de Normas Internacionales de Información Financiera (2011) a partir del año 2008 deben ser 
aplicadas en un $100 \%$ por las grandes empresas.

De acuerdo a revisión realizadas a las NIC y NIIF vigentes a diciembre de 2010 , el concepto de valor razonable se aplica a las diferentes partidas que integran los elementos de los estados financieros como son: activo, pasivo, patrimonio neto, ingresos y gastos. Al analizar la aplicación del valor razonable a cada partida de los elementos de los estados financieros, se observa que para algunas partidas del activo el método es aplicado al momento de la medición inicial, y para otras, es recomendado como un tratamiento alternativo, al momento de la medición posterior.

En resumen la normativa contable aplicada en Venezuela, referente al valor razonable corresponde a las mismas disposiciones establecidas en las NIC-NIIF.

\section{Comparabilidad de la información financiera bajo la medición del valor razonable}

Respecto a la comparabilidad de la información financiera presentada a valor razonable, De la Hoz, Uzcátegui y Borges (2008) afirman que este criterio contenido actualmente dentro del marco conceptual de las Normas Internacionales de Contabilidad (NIC) expresa que los usuarios deben ser capaces de comparar los estados financieros de una empresa a través del tiempo, a fin de identificar tendencias en la posición financiera y en sus resultados.

Explica Antonetti (2005) que la aplicación homogénea de índices determinan valores que no siempre tienen rela- ción con el valor de reposición depreciado de los bienes y producen resultados cuyas expresiones cuantitativas no son comparables entre sí. Afirma que una de las premisas fundamentales de cualquier información contable es que la misma sea comparable.

Fortis y García (2006) agregan que los usuarios de información financiera deben ser capaces de analizar los estados de una empresa a lo largo del tiempo, así como de compararlos con los de otros competidores, con el objeto de evaluar su posición financiera, desempeño y cambios en la posición financiera en términos relativos. Para ello, acontecimientos y situaciones similares deben ser tratados de un modo igual.

Mendoza (2008) afirma que existe un convencimiento generalizado de la doctrina contable internacional en relación a que la falta de homogenización y comparabilidad de información financiera, constituye el principal efecto de la coexistencia de diversos sistemas contables. Esto complica por una parte, la labor de las empresas que poseen filiales en distintos países con diferente normativa contable, y por la otra, se obstaculiza la toma de decisiones reflejado el acceso a mercados financieros.

Por tanto, la aplicación del valor razonable facilita la comparabilidad de la información financiera, lo cual es una característica cualitativa indispensable para estar en condiciones de utilizar la información en cualquier contexto económico, sea nacional o internacional, siendo relevante el uso de métodos que sean comparables entre si, eliminando las distorsiones potenciales que limitan la toma de decisiones. 
Valor Razonable como método de medición de la Información Financiera

Gómez, Oda; De La Hoz, Betty y López, Margo

\section{Ventajas y desventajas del valor razonable}

El uso del valor razonable está en correspondencia con las necesidades de reflejar en la información financiera el valor de la entidad. De acuerdo con Hervás (2003), existen algunas ventajas posibles de considerar al momento de aplicar el valor razonable en las operaciones de las empresas, entre las cuales incluye: da una señal al mercado de lo que realmente vale la empresa; mejora el principio de imagen fiel, mejorando el análisis financiero; permite la comparabilidad; procura información ajustada en cuanto a las depreciaciones y amortizaciones; los costos son calculados sobre bases reales y se estiman con exactitud las plusvalías en caso de venta.

Para Rojo (2001), al medir los activos y pasivos a valor razonable, suministra índices de rendimiento que reflejan la realidad de la organización, por lo que al presentar la información financiera a valores ajustados a la realidad, son de mayor utilidad para la toma de decisiones.

Según Pérez (2005), el valor razonable presenta varias ventajas como son: los elementos del patrimonio se valoran conforme al mercado, cumpliendo con la características de fiabilidad, lo cual favorece a la empresa en su capacidad para obtener más recursos, suministrando información más relevante para la toma de decisiones; la amortizaciones se calcularan sobre valores actualizados, recuperando más rápido el capital invertido y los costos se determinan sobre bases más reales. Así mismo, Madrigal Moreno (2010), menciona que la ventaja está dada por que refleja el valor real del activo.
Por otra parte, Rojo (2001) menciona que la desventaja del valor razonable es que su medición no es objetiva, ya que no hay medidas matemáticas para la determinación del valor. Mientras Hervás (2003), señala otros aspectos que pueden constituir desventajas visibles del uso del valor razonable, entre las cuales incluye: incremento de los gastos por registrar más depreciaciones y amortizaciones producto de revalorizaciones hechas en momentos no oportunos; los incrementos patrimoniales, pueden dar señal positiva a los accionistas (pago de dividendos), que limite la liquidez de la entidad; un aumento de las cifras en el balance puede ubicar a la empresa en otros niveles legales o fiscales que antes no alcanzaba; no pueden ser comparables con cifras al costo histórico y la empresa puede ser discriminada al momento de endeudarse pues sus indicadores de solvencia o rentabilidad al cambiar con las revalorizaciones infieren falsos cambios en su posición de solvencia, rentabilidad o relaciones costo-beneficio.

Para Pérez (2005) las desventajas están dadas por la falta de fiabilidad del valor razonable principalmente cuando no hay un mercado activo y se tienen que aplicar técnicas de valoración; así mismo puede originar costos de agencia por las asimetrías de información entre los que elaboran la información contable y sus usuarios.

También Madrigal (2010), menciona una serie de desventajas como: limitaciones para su cálculo en forma directa; depende de las condiciones volátiles del mercado; incidencia de los mercados globales; la valuación por medio de los flujos de efectivo futuro, son estimaciones; imprecisión en el cálculo, lo cual origina ele- 
vada incertidumbre; los métodos de cuantificación pueden ser variados $y$ complejos; y existe una estrecha interrelación entre diferentes eventos y circunstancias de una entidad.

Los argumentos antes expuestos en cuanto a las ventajas y desventajas que presenta el valor razonable indican que a nivel académico se debe continuar investigando sobre la validez de este método de medición, con el objeto de garantizar que los criterios de aplicación práctica reflejen en la información financiera la situación real de la entidad.

La gerencia de las organizaciones como responsable de la cuantificación y revelación de la información financiera a valor razonable, requiere conocer y asimilar el concepto de valor razonable y todas las disposiciones que se deben consideran para su cálculo y revelación, con el propósito de garantizar la fiabilidad de la información financiera.

\section{Conclusiones}

El valor razonable produjo un cambio de paradigma en la contabilidad financiera donde se le da prioridad a la esencia financiera que a la forma legal, permitiendo reflejar la realidad de los negocios, proporcionando mayor relevancia a la información ya que incorpora información actual y no referida a momentos pasados, siempre y cuando haya un mercado activo. También permite la comparabilidad de la información financiera
La principal crítica a este método de medición es la falta de objetividad; sin embargo, los estudios realizados bajo la supervisión de la SEC, a raíz de la crisis financiera en el año 2008, determinó que este método de medición no originó la caída de los mercados, su uso fue defendido por los inversores, ya que consideran que mejora la transparencia de la información financiera; a pesar que se considera que deben hacer mejoras.

En respuesta a lo antes indicado en mayo de 2011, el IASB emite la NIIF13. Medición del Valor Razonable, siendo necesario su estudio, asimilación y aplicación por parte de los responsables de preparar y examinar la información financiera de las organizaciones.

En resumen, el método de medición del valor razonable, tiene múltiples facetas, donde hay defensores y contrarios a su aplicación, siendo necesario estudiar las limitaciones detectadas por los preparadores de la información financiera al momento de aplicar el valor razonable en los diferentes elementos de los estados financieros, así como recoger la opinión de los usuarios de esta información, respecto a la contribución de tomar una decisión con base a información financiera a valor razonable. Para validar este método se requiere de investigación de campo, ya que hay dudas sobre sus beneficios y no hay evidencia clara que la información financiera a valor razonable, refleje la situación real del negocio. 
Valor Razonable como método de medición de la Información Financiera

Gómez, Oda; De La Hoz, Betty y López, Margo

\section{Referencias bibliográficas}

Antonetti, G. (2005). Experiencias de la aplicación de la reexpresión en las empresas. En: www.ave.org.ve/intranet/centro. Consultado el 15 de Julio de 2009 .

De la Hoz, Betty, Uzcátegui, Sigilfredo; Borges, Jesús y Velazco Ángel (2008). La inflación como factor distorsionante de la información financiera. Revista Venezolana de Gerencia. Vol. 13. No. 44, Dic. pp. 556-572.

Deloitte (2008) Adopción del valor razonable - Actualización Desarrollos y observaciones a partir de la adopción amplia del SFAS 157 y del SFAS159. Regulatory \& Capital Markets Consulting.

Federación de Colegio de Contadores Públicos de Venezuela (1997): Normas Básicas y Principios de Contabilidad de Aceptación General. Declaración de Principio de Contabilidad $N^{\circ} 0$ (DPC-0). Cabimas, Estado Zulia, Venezuela.

Federación de Colegio de Contadores Públicos de Venezuela (2011): Marco de Adopción de Normas Internacionales de Información Financiera. BA-VEN- NIF $\mathbf{N}^{\circ} \mathbf{0}$. Caracas, Distrito Capital.

Financial Accounting Standards Board (FASB) (2006) Fair Value Measurements SFAS No. 157. EE.UU.

Fortis, I. y García, I. (2006). La contabilidad según valor razonable. Centro de Estudios Monetarios Latinoamericanos. New York.

García, María y Zorio, Ana (2002). El Camino hacia el valor razonable. Revista de Contabilidad, vol. 5, num. 9.

Hervás Oliver (2003). Contabilidad, Inflación y Revalorizaciones: un tema pendiente en relación al fair value. Recono- cido a su vez por reconoce a su vez HITZ, Joerg-

International Accounting Standards Board (2005). Normas Internacionales de Información Financiera (NIIF).Inglaterra Londres. Traducción oficial al castellano por el CIIS de México.

International Accounting Standards Board (2006). Discussion Paper: Fair Value Measurements, Part 1: Invitation to Comment and relevant I.F.R.S. guidance, Londres.

International Accounting Standards Board (2006). Normas Internacionales de Información Financiera (NIIF) Londres.

International Accounting Standards Board (2009). Fair Value Measurement. Londres.

International Accounting Standards Board (2011). IFRS-13 Fair Value Measurement Londres

Madrigal Moreno, Franciso Javier (2010). Los conjuntos barrosos como propuesta a la complejidad para determiner el valor razonable en los activos biològicos. Universidad del Valle de Atemajac. Instituto Tecnològico de Estudios Superiores de Zamora. Mexico

Mantilla, Samuel Alberto (2009) Contabilidad a valor razonable. Colección Ciencias Administrativas. Área Contabilidad. Primera edición. Bogotá.

Mendoza, G. (2008). La Armonización de la Información Financiera y las Normas Internacionales de Contabilidad en los Agronegocios. Un Análisis Teórico Comparado. Universidad Católica del Táchira.

Pérez López, María Carmen (2005) La Influencia del valor razonable de los bienes inmuebles en el análisis de los estados financieros de la empresa Inmobiliaria. Tesis Doctoral de la Universidad de Granada 
Ryan Bob, Scapens Robert y Theobald Michael. (2004). Metodología de la Investigación en Finanzas $y$ Contabilidad. Ediciones Deusto. Planeta DeAgostini Profesional y Formación, S.L. Barcelona-España

Rojo, Alfonso (2001). Valoración contable, valor razonable y utilidad de la información. Revista de la Asociación Española de Contabilidad y Administración de Empresas No. 56. Especial XI Congreso AECA.
Securities and Exchange Commission (SEC) (2008). This is a report by the Staff of the U.S. Securities and Exchange Commission. The Commission has expressed no view regarding the analysis, findings, or conclusions contained herein, EE.UU.

Trombetta, Marco (2009). EI valor razonable no es la panacea de la contabilidad. IE Business School. 